\title{
ON THE NUMERICAL SOLUTION OF A TIME-DEPENDENT SHAPE OPTIMIZATION PROBLEM FOR THE HEAT EQUATION
}

\author{
RAHEL BRÜGGER*, HELMUT HARBRECHT*, AND JOHANNES TAUSCH ${ }^{\dagger}$
}

\begin{abstract}
This article is concerned with the solution of a time-dependent shape identification problem. Specifically we consider the heat equation in a domain, which contains a time-dependent inclusion of zero temperature. The objective is to detect this inclusion from the given temperature and heat flux at the exterior boundary of the domain. To this end, for a given temperature at the exterior boundary, the mismatch of the Neumann data is minimized. This time-dependent shape optimization problem is then solved by a gradient-based optimization method. Numerical results are presented which validate the present approach.
\end{abstract}

Key words. Inverse problem, shape optimization, heat equation

1. Introduction. Shape optimization appears in a wide range of problems from engineering, especially for designing and constructing industrial components or in non-destructive testing. Many practical problems from engineering amount to partial differential equations for an unknown function, which needs to be computed to obtain the quantity of interest. Shape optimization is then concerned with the minimization of this quantity of interest. While shape optimization in case of elliptic partial differential equations is a well studied topic in literature, see for example [5, 27] and the references therein, not so much is known about shape optimization in case of parabolic partial differential equations.

Theoretical results for parabolic shape optimization problems with time-independent shapes can be found in [26, 27, 32], while practical results are found for example in $[2,3,13]$. This is in contrast to the results for parabolic shape optimization problems with time-dependent shapes. Theoretical results are for example available in [7, 8, 23], but to the best of our knowledge, no results about efficient computations of such timedependent shape optimization problems exist.

This article is based on the previous article [13] by two of the authors, where a parabolic shape optimization problem is considered for a time-independent shape. The goal therein was to detect a fixed inclusion or void of zero temperature inside a three-dimensional solid or liquid body by measurements of the temperature and the transient heat flux at the accessible outer boundary. Since the underlying shape calculus turned out to be rather standard due to the stationarity of the inclusion, the focus has been on the development of an efficient solver for the underlying heat equation. In contrast, in the present article, we now consider an inclusion, which changes its shape during time. Therefore, the shape calculus becomes the focus, while the numerical experiments are performed in two space dimensions and serve as a proof of concept.

The problem under consideration is reformulated as a shape optimization problem by means of a tracking-type functional for the Neumann data. Therefore, for given temperature at the exterior boundary, the mismatch of the Neumann data is minimized in a least-squares sense. Since we intend to apply a gradient-based optimization algorithm, we compute the shape gradient of this functional by means of the adjoint approach, which is known to reduce the computational effort. Then, we make a parametric ansatz for the inclusion and use a boundary element method to

\footnotetext{
*Departement Mathematik und Informatik, Universität Basel, Spiegelgasse 1, 4051 Basel, Schweiz, \{ra.bruegger,helmut.harbrecht\}@unibas.ch

${ }^{\dagger}$ Department of Mathematics, Southern Methodist University, Dallas, TX 75275, tausch@smu.edu
} 
solve the heat equations for the primal state and the adjoint state. Numerical results validate that the present approach is feasible, leading to meaningful reconstructions.

The remainder of the article is organized as follows. In Section 2, we state the problem under consideration. Section 3 is dedicated to the time-dependent shape calculus of our functional. Section 4 shows how we can discretize our problem in the case of a void which is star-shaped for all points of time. In order to solve the heat equation on the current domain, Section 5 explains how to do this by using a boundary element method. Since the method parallels that of [13], this section only discusses the changes for the moving boundaries considered in this article. In order to illustrate the developed techniques, they are applied to the examples shown in Section 6. Finally, in Section 7, we give some concluding remarks.

\section{Problem formulation.}

2.1. Model problem. Let $D \subset \mathbb{R}^{d}$ with $d=2,3$ be a simply connected, spatial domain with boundary $\Gamma^{f}=\partial D$. Moreover, we have a time component, and thus the domain $(0, T) \times D$ forms a cylindrical domain, called the space-time cylinder. At every time $t \in[0, T]$, a simply connected subdomain $S_{t} \subset D$ with boundary $\Gamma_{t}=\partial S_{t}$ lies inside $D$ such that it holds $\operatorname{dist}\left(\Gamma^{f}, \Gamma_{t}\right)>0$ for all $t$. The difference domain is called $\Omega_{t}:=D \backslash \overline{S_{t}}$. Taking into account the time again, we thus consider tubes (i.e., non-cylindrical domains), which contain a void and are represented as

$$
Q_{T}=\bigcup_{0<t<T}\left(\{t\} \times \Omega_{t}\right) .
$$

The interior boundary of the tube $Q_{T}$ is called

$$
\Sigma_{T}=\bigcup_{0<t<T}\left(\{t\} \times \Gamma_{t}\right)
$$

and the exterior boundary of the tube is called $\Sigma^{f}=(0, T) \times \Gamma^{f}{ }^{1}$ The topological setup is illustrated in Figure 2.1. It is in analogy to [13], but we consider an interior boundary $\Gamma_{t}$ which moves in time instead of a fixed, interior boundary $\Gamma_{0}$.

For every point of time $t$, we assume to have a smooth $C^{2}$-diffeomorphism $\kappa$, which maps the initial domain $\Omega_{0}$ onto the time-dependent domain $\Omega_{t}$. In accordance with [23], we write

$$
\boldsymbol{\kappa}:[0, T] \times \mathbb{R}^{d} \rightarrow \mathbb{R}^{d}, \quad(t, \mathbf{x}) \mapsto \boldsymbol{\kappa}(t, \mathbf{x})
$$

to emphasize the dependence of the mapping $\boldsymbol{\kappa}$ on the time, where we have $\boldsymbol{\kappa}\left(t, \Omega_{0}\right)=$ $\Omega_{t}$. Here, $\kappa \in C^{2}\left([0, T] \times \mathbb{R}^{d}\right)$ and, as in [12, pg. 826], we assume the uniformity condition

$$
\|\boldsymbol{\kappa}(t, \mathbf{x})\|_{C^{2}\left([0, T] \times \mathbb{R}^{d} ; \mathbb{R}^{d}\right)},\left\|\boldsymbol{\kappa}(t, \mathbf{x})^{-1}\right\|_{C^{2}\left([0, T] \times \mathbb{R}^{d} ; \mathbb{R}^{d}\right)} \leq C_{\boldsymbol{\kappa}}
$$

for some constant $C_{\boldsymbol{\kappa}} \in(0, \infty)$. To reduce the technical level of the ensuing discussion, we assume that $\Omega_{0}$ has $C^{2}$-smooth boundaries which implies that the boundaries of $\Omega_{t}$ have the same regularity. [12]

REMARK 2.1. Notice that, due to the uniformity condition (2.2), we have as in

$$
0<\underline{\sigma} \leq \min \{\sigma(\mathrm{D} \boldsymbol{\kappa})\} \leq \max \{\sigma(\mathrm{D} \boldsymbol{\kappa})\} \leq \bar{\sigma}<\infty,
$$

\footnotetext{
${ }^{1}$ We assume that the exterior boundary $\Gamma^{f}$ does not depend on time, but this is no necessity for the shape calculus presented in the subsequent chapter.
} 


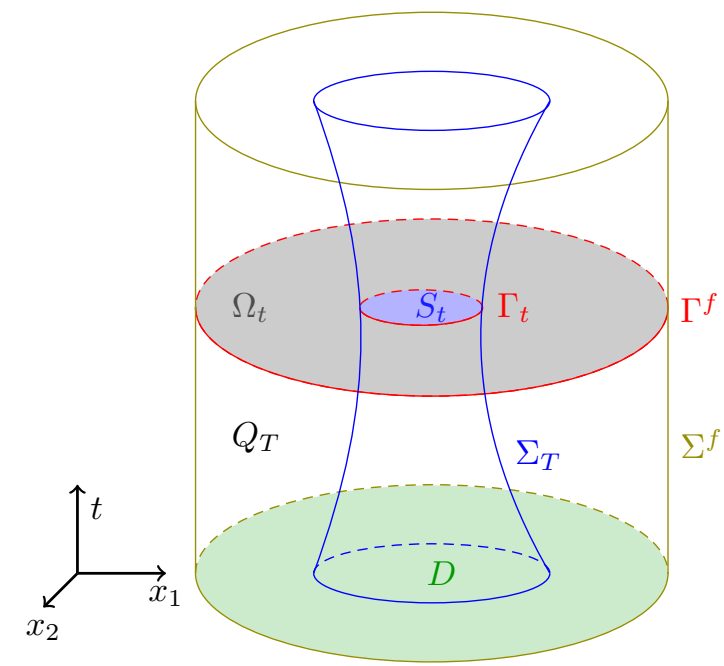

Figure 2.1: The tube $Q_{T}$ with the boundaries $\Sigma_{T}$ and $\Sigma^{f}$ for $d=2$.

where $\sigma($.$) denote the singular values and \mathrm{D} \boldsymbol{\kappa}$ denotes the Jacobian matrix of $\boldsymbol{\kappa}$. Moreover, as in [12, Remark 1, pg. 827], we assume $\operatorname{det}(\mathrm{D} \kappa)$ to be positive. The smoothness of the mapping also implies that the time derivative $\partial_{t} \kappa$ is uniformly bounded.

We shall consider the following, overdetermined initial boundary value problem for the heat equation, where $f$ and $g$ are defined at the fixed exterior boundary $\Sigma^{f}$

$$
\begin{array}{cc}
\partial_{t} u=\Delta u & \text { in } Q_{T}, \\
u=0 & \text { on } \Sigma_{T}, \\
u=f, \frac{\partial u}{\partial \mathbf{n}}=g & \text { on } \Sigma^{f}, \\
u(0, \cdot)=0 & \text { in } \Omega_{0} .
\end{array}
$$

Here, $\mathbf{n}$ denotes the normal pointing outward of the domain $\Omega_{t}$. In what follows, we assume that $f$ vanishes for $t=0$, which implies the compatibility with the initial condition. We then seek the free boundary $\Sigma_{T}$, such that the overdetermined problem (2.3) allows for a solution $u$. In [2, Theorem 1.1], the uniqueness of such a boundary $\Sigma_{T}$ is proven in the case of a time-independent boundary. The uniqueness in the timedependent case of such an inverse problem is stated in [16, Proposition 3.1], subject to certain conditions on the sought-after domain.

2.2. Reformulation as a shape optimization problem. The task of finding the unknown boundary $\Sigma_{T}$ is reformulated as a shape optimization problem by introducing the function $v$ as the solution of the initial boundary value problem with Dirichlet boundary conditions for the heat equation

$$
\begin{aligned}
\partial_{t} v & =\Delta v & & \text { in } Q_{T}, \\
v & =0 & & \text { on } \Sigma_{T}, \\
v & =f & & \text { on } \Sigma^{f}, \\
v(0, \cdot) & =0 & & \text { in } \Omega_{0} .
\end{aligned}
$$


We set $Q_{0}=(0, T) \times \Omega_{0}$, which has two time-independent boundaries denoted by $\Sigma_{0}:=(0, T) \times \partial \Omega_{0}$. The appropriate function spaces for parabolic problems in time invariant domains are the anisotropic Sobolev spaces, defined by

$$
H^{r, s}\left(Q_{0}\right):=L^{2}\left((0, T) ; H^{r}\left(\Omega_{0}\right)\right) \cap H^{s}\left((0, T) ; L^{2}\left(\Omega_{0}\right)\right)
$$

for $r, s \in \mathbb{R}_{\geq 0}$, see, e.g., $[2,4,21]$. Likewise, the corresponding boundary spaces are

$$
H^{r, s}\left(\Sigma_{0}\right):=L^{2}\left((0, T) ; H^{r}\left(\Sigma_{0}\right)\right) \cap H^{s}\left((0, T) ; L^{2}\left(\Sigma_{0}\right)\right)
$$

which are defined for $C^{2}$-boundary when $r \leq 2$. With these definitions at hand, we can moreover define

$$
\begin{aligned}
& \hat{H}^{r, s}\left(Q_{0}\right):=\left\{u=\left.U\right|_{Q_{0}}: U \in H^{r, s}\left((-\infty, T) \times \Omega_{0}\right), U(t, \cdot)=0, t<0\right\}, \\
& \tilde{H}^{r, s}\left(Q_{0}\right):=\left\{u=\left.U\right|_{Q_{0}}: U \in H^{r, s}\left((0, \infty) \times \Omega_{0}\right), U(t, \cdot)=0, T<t\right\}, \\
& \hat{H}^{r, s}\left(\Sigma_{0}\right):=\left\{u=\left.U\right|_{\Sigma_{0}}: U \in H^{r, s}\left((-\infty, T) \times \Sigma_{0}\right), U(t, \cdot)=0, t<0\right\} .
\end{aligned}
$$

As in the elliptic case, we can include also (spatial) zero boundary conditions into the function spaces by setting

$$
\begin{aligned}
& \hat{H}_{0}^{r, s}\left(Q_{0}\right):=\left\{u \in \hat{H}^{r, s}\left(Q_{0}\right):\left.u\right|_{\Sigma_{0}}=0\right\}, \\
& \tilde{H}_{0}^{r, s}\left(Q_{0}\right):=\left\{u \in \tilde{H}^{r, s}\left(Q_{0}\right):\left.u\right|_{\Sigma_{0}}=0\right\} .
\end{aligned}
$$

The dual spaces are denoted by $r, s \leq 0$ and we especially have

$$
\hat{H}^{r, s}\left(Q_{0}\right)=\left[\tilde{H}_{0}^{-r,-s}\left(Q_{0}\right)\right]^{\prime} \text { for } r-\frac{1}{2} \notin \mathbb{Z} .
$$

We are now in the position to introduce the non-cylindrical analogues of the above spaces by setting

$$
H^{r, s}\left(Q_{T}\right):=\left\{v \in L^{2}\left(Q_{T}\right): v \circ \kappa \in H^{r, s}\left(Q_{0}\right)\right\},
$$

where the composition with $\boldsymbol{\kappa}$ only acts on the spatial component. Due to the chain rule, $v \circ \boldsymbol{\kappa}$ and $v$ have the same Sobolev regularity, provided that the mapping $\boldsymbol{\kappa}$ is smooth enough, see for example [22, Theorem 3.23] for the elliptic case. We especially have the equivalence of norms for $|s| \leq 2$

$$
\|v \circ \boldsymbol{\kappa}(t, \cdot)\|_{H^{s}\left(\Omega_{0}\right)} \sim\|v(t, \cdot)\|_{H^{s}\left(\Omega_{t}\right)} .
$$

For the cylindrical case it is well known that the solution operator $f \mapsto S_{0} f:=u$ of the Dirichlet problem of the heat equation

$$
\begin{array}{rlr}
\left(\partial_{t}-\Delta\right) u=0 & & \text { in } Q_{0}, \\
u=f & & \text { on } \Sigma_{0},
\end{array}
$$

with homogeneous initial conditions is an isomorphism between the spaces

$$
S_{0}: \hat{H}^{\frac{1}{2}+s,\left(\frac{1}{2}+s\right) / 2}\left(\Sigma_{0}\right) \rightarrow \hat{H}^{1+s,(1+s) / 2}\left(Q_{0}\right)
$$

for $s>-\frac{1}{2}$ when $\Omega_{0}$ is smooth and for $|s|<\frac{1}{2}$ when $\Omega_{0}$ is Lipschitz, see [21, Theorem 5.3] and [4, Proposition 4.13]. 
For the existence, uniqueness and regularity of solutions to (2.4), we have to make sure the analogous result also holds on a non-cylindrical domain. The main technique of the argument is to transport the heat equation to a parabolic problem with variable coefficients in the space-time cylinder $Q_{0}$ and apply the same functional analytic tools of the above references there.

THEOREM 2.2. There exists a unique solution $v \in \hat{H}^{1, \frac{1}{2}}\left(Q_{T}\right)$ satisfying the boundary condition in (2.4) and

$$
S(v, \varphi):=\int_{0}^{T} \int_{\Omega_{t}}\left\{\nabla v \cdot \nabla \varphi+\partial_{t} v \varphi\right\} \mathrm{d} \mathbf{x} \mathrm{d} t=0 \text { for all } \varphi \in \tilde{H}_{0}^{1, \frac{1}{2}}\left(Q_{T}\right) .
$$

Proof. The assertion follows if we can show existence and uniqueness of the solution to the following generalization of problem (2.4)

$$
\begin{aligned}
\left(\partial_{t}-\Delta\right) v=q & & \text { in } Q_{T}, \\
v=f & & \text { on } \Sigma_{T} \cup \Sigma^{f}, \\
v(0, \cdot)=0 & & \text { in } \Omega_{0},
\end{aligned}
$$

where $f \in \hat{H}^{\frac{1}{2}, \frac{1}{4}}$ and $q \in \hat{H}^{-1,-\frac{1}{2}}\left(Q_{T}\right)$. Its weak formulation reads

$$
S(v, u)=\int_{0}^{T} \int_{\Omega_{t}} q u \mathrm{~d} \mathbf{x} \mathrm{d} t
$$

where $S$ is given by (2.5). We set $u^{t}=u \circ \boldsymbol{\kappa}$ and similarly for $v^{t}$ and $q^{t}$.

We first show the analogue of $[4$, Lemma 2.3]: Let $f=0$. For every $q \in$ $L^{2}\left((0, T) ; H^{-1}\left(\Omega_{t}\right)\right)$, there exists a unique solution $v \in \mathcal{V}_{0}\left(Q_{T}\right)$ of (2.6), where the space $\mathcal{V}_{0}\left(Q_{T}\right)$ consists of all the functions $v$ with $v \circ \kappa \in \mathcal{V}_{0}\left(Q_{0}\right)$ and

$$
\mathcal{V}_{0}\left(Q_{0}\right):=\left\{u \in L^{2}\left((0, T) ; H_{0}^{1}\left(\Omega_{0}\right)\right): \partial_{t} u \in L^{2}\left((0, T) ; H^{-1}\left(\Omega_{0}\right)\right)\right\} .
$$

Notice that this space is a dense subspace of $H_{0}^{1, \frac{1}{2}}\left(Q_{0}\right)$ and coincides with the space $W(0, T)$ from [31, Definition 25.3].

Transforming (2.7) back to $Q_{0}$ by using Lemma A.2 with $\boldsymbol{\xi}=\boldsymbol{\kappa}, Q_{\varsigma}=Q_{T}$ and $Q_{\tau}=Q_{0}$ gives

$$
\int_{0}^{T}\left\langle\partial_{t} v^{t}(t), u^{t}(t)\right\rangle_{L^{2}\left(\Omega_{0}\right)}+a\left(t ; v^{t}(t), u^{t}(t)\right) \mathrm{d} t=\int_{0}^{T}\left\langle q^{t}(t), u^{t}(t)\right\rangle_{L^{2}\left(\Omega_{0}\right)} \mathrm{d} t,
$$

where $a$ is defined in Lemma A.2.

To show solvabilty of (2.7) we apply [20, Chapter 3, Theorem 4.1] or [31, Theorem 26.1], which require boundedness and coercivity of $a$. The boundedness follows easily from Remark 2.1. It remains to show coercivity, that is, there exist some constants $\alpha>0, \lambda \in \mathbb{R}$, such that for almost all $t \in(0, T)$

$$
a\left(t ; u^{t}, u^{t}\right) \geq \alpha\left\|u^{t}\right\|_{H^{1}\left(\Omega_{0}\right)}^{2}-\lambda\left\|u^{t}\right\|_{L^{2}\left(\Omega_{0}\right)}^{2}
$$

holds for all $u^{t} \in H_{0}^{1}\left(\Omega_{0}\right)$. With the help of the Cauchy-Schwarz inequality, we have

$$
\begin{aligned}
a\left(t ; u^{t}, u^{t}\right) \geq & \int_{\Omega_{0}}\left\|(\mathrm{D} \boldsymbol{\kappa})^{-\boldsymbol{\top}} \nabla u^{t}\right\|^{2} \mathrm{~d} \mathbf{x} \\
& -\int_{\Omega_{0}}\left\|(\underbrace{(\mathrm{D} \boldsymbol{\kappa})^{-\boldsymbol{\top}} \frac{1}{\operatorname{det}(\mathrm{D} \boldsymbol{\kappa})} \nabla(\operatorname{det}(\mathrm{D} \boldsymbol{\kappa}))}_{:=a_{1}}+\underbrace{\partial_{t} \boldsymbol{\kappa}}_{:=a_{2}}) u^{t}\right\|\left\|(\mathrm{D} \boldsymbol{\kappa})^{-\boldsymbol{\top}} \nabla u^{t}\right\| \mathrm{d} \mathbf{x} .
\end{aligned}
$$


Completing the square gives

$$
\begin{aligned}
a\left(t ; u^{t}, u^{t}\right) & \geq \underbrace{\int_{\Omega_{0}} \frac{1}{2}\left(\left\|(\mathrm{D} \boldsymbol{\kappa})^{-\mathrm{T}} \nabla u^{t}\right\|-\left\|\left(a_{1}+a_{2}\right) u^{t}\right\|\right)^{2}}_{\geq 0} \mathrm{~d} \mathbf{x} \\
& +\int_{\Omega_{0}} \frac{1}{2}\left\|(\mathrm{D} \boldsymbol{\kappa})^{-\mathrm{T}} \nabla u^{t}\right\|^{2} \mathrm{~d} \mathbf{x}-\int_{\Omega_{0}} \frac{1}{2}\left\|\left(a_{1}+a_{2}\right) u^{t}\right\|^{2} \mathrm{~d} \mathbf{x} .
\end{aligned}
$$

Discarding the positive term and due to Remark 2.1, we have

$$
a\left(t ; u^{t}, u^{t}\right) \geq C\left|u^{t}\right|_{H^{1}\left(\Omega_{0}\right)}^{2}-\frac{1}{2} \int_{\Omega_{0}}\left|u^{t}\right|^{2}\left\|a_{1}+a_{2}\right\|^{2} \mathrm{~d} \mathbf{x}
$$

and, therefore, by using the parallelogram law

$$
a\left(t ; u^{t}, u^{t}\right) \geq C\left|u^{t}\right|_{H^{1}\left(\Omega_{0}\right)}^{2}-\int_{\Omega_{0}}\left|u^{t}\right|^{2}\left(\left\|a_{1}\right\|^{2}+\left\|a_{2}\right\|^{2}\right) \mathrm{d} \mathbf{x} .
$$

Now we can apply again Remark 2.1 to $a_{1}$ and $a_{2}$ and the Poincaré-Friedrichs inequality to the first term to arrive at the desired estimate (2.8).

Secondly, following the lines of [4], the analogue of [4, Lemma 2.8] reads: For every $q \in \hat{H}^{-1,-\frac{1}{2}}\left(Q_{T}\right)$, there exists a unique $v \in \hat{H}_{0}^{1, \frac{1}{2}}\left(Q_{T}\right)$ satisfying $\left(\partial_{t}-\Delta\right) v=q$ in $Q_{T}$. For the proof, we can straightforwardly modify the proof [4, Lemma 2.8], which is based on adjoint operators and interpolation results. The interpolation results also hold on the spaces with respect to the tube $Q_{T}$ and the adjoint operators with respect to $Q_{T}$ have the same structure as the adjoint operators in [4] with respect to $Q_{0}$.

Thirdly, due to the surjectivity of the trace operator, we can then follow the proof of $[4$, Theorem 2.9] to obtain the statement in the theorem.

REMARK 2.3. If the Dirichlet data in (2.4) satisfy $f \in \hat{H}^{\frac{3}{2}}, \frac{3}{4}\left(\Sigma^{f}\right)$, then the solution $v$ of (2.5) lies in $\hat{H}^{2,1}\left(Q_{T}\right)$. This is a consequence of [18, Chapter IV, Theorem 9.1].

For the given state equation (2.4), we introduce the tracking-type functional for the Neumann data at the fixed boundary $\Sigma^{f}$

$$
J\left(\Sigma_{T}\right)=\frac{1}{2} \int_{0}^{T} \int_{\Gamma^{f}}\left(\frac{\partial v}{\partial \mathbf{n}}-g\right)^{2} \mathrm{~d} \sigma \mathrm{d} t .
$$

This objective functional should be minimized in the space of admissible boundaries $\Sigma_{T}$. It is nonnegative, and it is zero and hence minimal if and only if $v=u$. The objective functional measures the $L^{2}$-error of the data mismatch and thus corresponds to the minimization in the least-squares sense. Notice that the existence of optimal solutions to the shape functional (2.9) can be proven by the techniques provided, for example, in $[1,17]$.

\section{Computation of the shape derivative.}

3.1. Shape calculus. In order to minimize the objective functional (2.9), we apply a gradient-based optimization method. To this end, we shall compute the shape derivative of the functional.

The shape calculus for time-dependent problems has been formulated by means of the speed method in [8] and [23]. The speed method allows for deformations which 
are not only small perturbations of the domain. One intends to find a velocity field $\mathbf{V}$, which generates the optimal tube. The solution $\mathbf{T}(t, \cdot): \mathbf{x} \mapsto \mathbf{x}_{t}=\mathbf{T}(t, \mathbf{x})$ of the differential equation [33, pg. 6$]$

$$
\begin{aligned}
\frac{\partial}{\partial t} \mathbf{T}(t, \mathbf{x}) & =\mathbf{V}(t, \mathbf{T}(t, \mathbf{x})) & & \text { in }(0, T) \times \Omega_{0}, \\
\mathbf{T}(0, \mathbf{x}) & =\mathbf{x} & & \text { in } \Omega_{0}
\end{aligned}
$$

describes the pathline of an individual particle being exposed to the velocity field $\mathbf{V}$. Hence, if we would inject a drop of dye at a certain point and time, and we do a timelapse photography, we would see the pathline [28]. In other words, when considering $t$ as the trajectory parameter, a fixed point $\mathbf{x}$ gets moved along the trajectory $\mathbf{x}_{t}=$ $\mathbf{T}(t, \mathbf{x})$. The point $\mathbf{x}$ can be thought of as the Lagrangian (or material) coordinate, while $\mathbf{x}_{t}$ is the Eulerian (field) coordinate [27, pg. 49]. The speed method is favorable when considering the Eulerian setting [23].

For the Lagrangian setting, which we consider here, the perturbation of identity is preferable. The shape calculus for the perturbation of identity is briefly stated in [23] as well. For our computations, we shall exploit the bijective mapping $\boldsymbol{\kappa}$ from (2.1), which implies the mapping scheme displayed in Figure 3.1. With the mapping $\boldsymbol{\kappa}$ we can associate the velocity field

$$
\mathbf{V}=\partial_{t} \boldsymbol{\kappa} \circ \boldsymbol{\kappa}^{-1},
$$

which could be used for the speed method. Since the outer boundary $\Sigma^{f}$ of the tube is fixed, this vector field is zero in normal direction there.

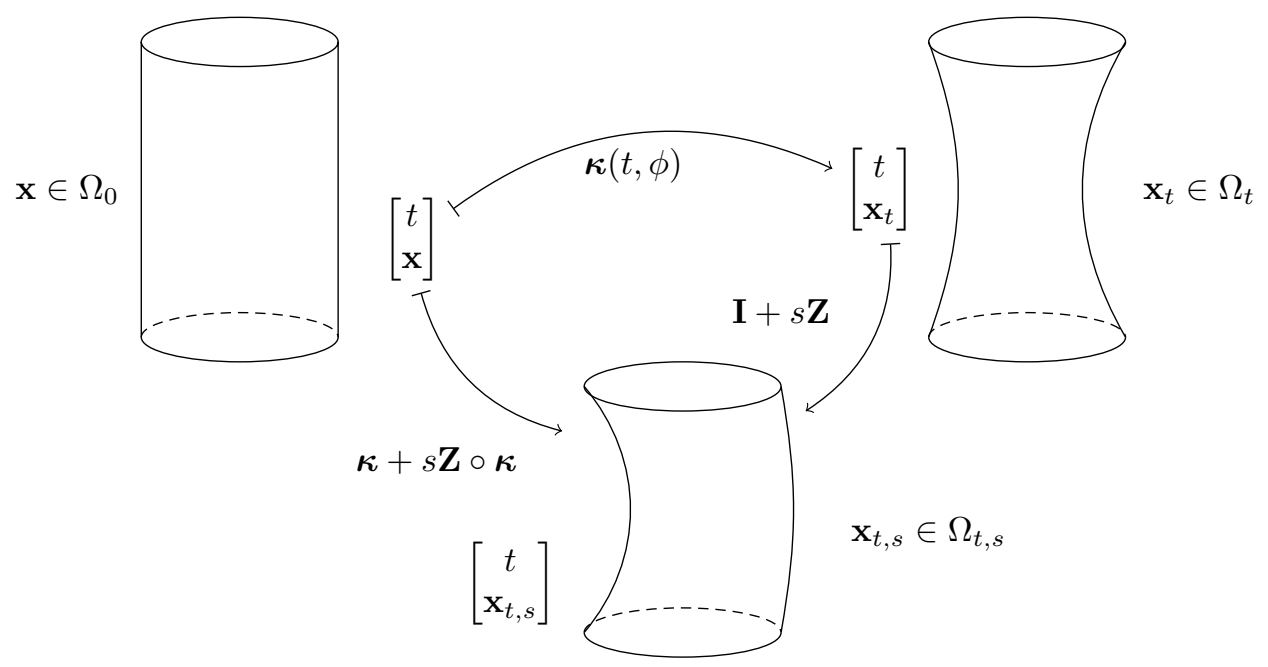

Figure 3.1: Perturbation of identity in the Lagrangian setting.

In order to apply the traditional shape calculus, we would like to perturb the tube. To this end, we consider a vector field $\mathbf{Z}(t, \mathbf{x})$, which generates the perturbation of identity $\mathbf{I}+s \mathbf{Z}$. It yields a new tube

$$
Q_{T}^{s}=\bigcup_{0<t<T}\left(\{t\} \times(\mathbf{I}+s \mathbf{Z})\left(\Omega_{t}\right)\right) .
$$


Notice that the perturbations under consideration are horizontal, meaning that we consider perturbations of $(t, \boldsymbol{\kappa})$ of the type $(0, \mathbf{Z})$, compare [23]. Moreover, $\mathbf{I}+s \mathbf{Z}$ should satisfy a uniformity condition as in (2.2).

3.2. Local shape derivative. Let us define the space of admissible perturbation fields as

$$
\mathcal{Z}_{\text {ad }}:=\left\{\mathbf{Z} \in C^{2}((0, T) \times D) \text { with } \mathbf{Z}=0 \text { in a neighbourhood of } \Sigma^{f}\right\}
$$

and consider a perturbation field $\mathbf{Z} \in \mathcal{Z}_{\text {ad }}$. As in the time-independent case, we can define non-cylindrical material and local shape derivatives. The material derivative $\dot{v}[\mathbf{Z}]$ is defined as

$$
\dot{v}[\mathbf{Z}]=\lim _{s \rightarrow 0} \frac{v_{t, s} \circ(\mathbf{I}+s \mathbf{Z})-v_{t}}{s},
$$

while the local shape derivative $\delta v=\delta v[\mathbf{Z}]$ in the direction $\mathbf{Z}$ is formally given by

$$
\delta v[\mathbf{Z}](t, \mathbf{x})=\lim _{s \rightarrow 0} \frac{v_{t, s}(t, \mathbf{x})-v_{t}(t, \mathbf{x})}{s}, \quad(t, \mathbf{x}) \in Q_{T}^{s} \cap Q_{T} .
$$

Here, $v_{t, s}$ denotes the state computed on the perturbed domain $Q_{T}^{s}$ and $v_{t}$ the state computed on $Q_{T}$, see [23, pg. 166]. These two non-cylindrical derivatives are connected by the relation

$$
\delta v[\mathbf{Z}]=\dot{v}[\mathbf{Z}]-\nabla v \cdot \mathbf{Z} .
$$

THEOREM 3.1. The local shape derivative of the state $v$ from (2.4) in the direction $\mathbf{Z} \in \mathcal{Z}_{\text {ad }}$ can be computed as the solution of the partial differential equation

$$
\begin{aligned}
\partial_{t} \delta v & =\Delta \delta v & & \text { in } Q_{T}, \\
\delta v & =-\langle\mathbf{Z}, \mathbf{n}\rangle \frac{\partial v}{\partial \mathbf{n}} & & \text { on } \Sigma_{T}, \\
\delta v & =0 & & \text { on } \Sigma^{f}, \\
\delta v(0, \cdot) & =0 & & \text { in } \Omega_{0} .
\end{aligned}
$$

The proof of the local shape derivative is presented in Appendix A, where we reformulate the time-independent proof found in [2] for the time-dependent setting. Notice that we consider $\mathbf{Z} \in \mathcal{Z}_{\text {ad }}$ to ensure that the mapping $\mathbf{I}+s \mathbf{Z}$ and its inverse can satisfy a uniformity condition analogous to (2.2) for $s$ small enough.

3.3. Shape derivative of the objective functional. With the local shape derivative at hand, we are in the position to compute the shape derivative of the objective functional (2.9), which is defined by

$$
\nabla J\left(Q_{T}\right)[\mathbf{Z}]=\lim _{s \rightarrow 0} \frac{J\left(Q_{T}^{s}\right)-J\left(Q_{T}\right)}{s} .
$$

THEOREM 3.2. The shape derivative of the objective functional (2.9) in the direction $\mathbf{Z} \in \mathcal{Z}_{\text {ad }}$ reads

$$
\nabla J\left(Q_{T}\right)[\mathbf{Z}]=-\int_{0}^{T} \int_{\Gamma_{t}} \frac{\partial p}{\partial \mathbf{n}} \frac{\partial v}{\partial \mathbf{n}}\langle\mathbf{Z}, \mathbf{n}\rangle \mathrm{d} \sigma \mathrm{d} t,
$$


where the adjoint state $p$ satisfies also the heat equation, but reversed in time:

$$
\begin{aligned}
-\partial_{t} p & =\Delta p & & \text { in } Q_{T}, \\
p & =0 & & \text { on } \Sigma_{T}, \\
p & =\frac{\partial v}{\partial \mathbf{n}}-g & & \text { on } \Sigma^{f}, \\
p(T, \cdot) & =0 & & \text { in } \Omega_{T} .
\end{aligned}
$$

Remark 3.3. According to Remark 2.3, we have $\frac{\partial v}{\partial \mathbf{n}} \in H^{\frac{1}{2}}, \frac{1}{4}\left(\Sigma^{f}\right)$. Hence, assuming also that $g \in H^{\frac{1}{2}}, \frac{1}{4}\left(\Sigma^{f}\right)$, the integrand of the functional $(2.9)$ is well-defined and also the adjoint problem (3.6) is well-defined, allowing for a solution $p \in \tilde{H}^{1, \frac{1}{2}}\left(Q_{T}\right)$. Therefore, the Neumann trace $\frac{\partial p}{\partial \mathbf{n}}$ lies in $H^{-\frac{1}{2},-\frac{1}{4}}\left(\Sigma_{T}\right)$. Together with the smoothness of $\mathbf{Z}$ and the smoothness of the domain under consideration, this yields a well-defined shape derivative (3.5).

Proof of Theorem 3.2. Due to $\mathbf{Z}=\mathbf{0}$ in a neighbourhood of $\Sigma^{f}$, we conclude

$$
\nabla J\left(Q_{T}\right)[\mathbf{Z}]=\int_{0}^{T} \int_{\Gamma^{f}} \frac{\partial \delta v}{\partial \mathbf{n}}\left(\frac{\partial v}{\partial \mathbf{n}}-g\right) \mathrm{d} \sigma \mathrm{d} t
$$

In view of the adjoint state equation (3.6), we can reformulate the derivative of $J$ by

$$
\nabla J\left(Q_{T}\right)[\mathbf{Z}]=\int_{0}^{T} \int_{\Gamma^{f}} p \frac{\partial \delta v}{\partial \mathbf{n}} \mathrm{d} \sigma \mathrm{d} t .
$$

To derive (3.5), we apply Green's theorem and obtain

$$
\begin{aligned}
0 & =\int_{0}^{T} \int_{\Omega_{t}}\left\{\left(\partial_{t} \delta v-\Delta \delta v\right) p+\delta v\left(\partial_{t} p+\Delta p\right)\right\} \mathrm{d} \mathbf{x} \mathrm{d} t \\
& =\int_{0}^{T} \int_{\Omega_{t}} \partial_{t}(\delta v p) \mathrm{d} \mathbf{x} \mathrm{d} t+\int_{0}^{T} \int_{\Gamma_{t} \cup \Gamma^{f}}\left\{\frac{\partial p}{\partial \mathbf{n}} \delta v-\frac{\partial \delta v}{\partial \mathbf{n}} p\right\} \mathrm{d} \sigma \mathrm{d} t
\end{aligned}
$$

Since the integrands are smooth enough, we can apply the Reynolds transport theorem (see [11, pg. 78] for example) to treat the domain integral. Recall that the velocity field $\mathbf{V}$, which transports the initial domain through the space-time tube, is given by (3.1). In combination with the end and initial conditions of $p$ and $\delta v$, respectively, we thus obtain

$$
\begin{aligned}
0=\underbrace{\int_{0}^{T} \frac{\mathrm{d}}{\mathrm{d} t} \int_{\Omega_{t}} \delta v p \mathrm{~d} \mathbf{x} \mathrm{d} t}_{=0}-\int_{0}^{T} \int_{\Gamma^{f} \cup \Gamma_{t}} & \underbrace{\delta v}_{=0 \text { on } \Gamma^{f}} \underbrace{p}_{=0 \text { on } \Gamma_{t}}\langle\mathbf{V}, \mathbf{n}\rangle \mathrm{d} \sigma \mathrm{d} t \\
& +\int_{0}^{T} \int_{\Gamma_{t}} \frac{\partial p}{\partial \mathbf{n}} \delta v \mathrm{~d} \sigma \mathrm{d} t-\int_{0}^{T} \int_{\Gamma^{f}} \frac{\partial \delta v}{\partial \mathbf{n}} p \mathrm{~d} \sigma \mathrm{d} t
\end{aligned}
$$

that is

$$
\int_{0}^{T} \int_{\Gamma^{f}} \frac{\partial \delta v}{\partial \mathbf{n}} p \mathrm{~d} \sigma \mathrm{d} t=\int_{0}^{T} \int_{\Gamma_{t}} \frac{\partial p}{\partial \mathbf{n}} \delta v \mathrm{~d} \sigma \mathrm{d} t
$$

Hence, by inserting the boundary condition for $\delta v$ as stated in (3.4), we finally arrive at the desired result (3.5). 
Note that the tracking-type functional for the Dirichlet data has been considered in the setting of the speed method in [23, pg. 36-46]. It leads also to the same local shape derivative and shape gradient as in the time-independent case derived in [13]. This is thus consistent with the formulae stated here in case of the tracking-type functional for the Neumann data.

REMARK 3.4. As one can see from Theorem 3.1, only the normal component of the perturbation field $\mathbf{Z}$ on $\Sigma_{T}$ is relevant. Therefore, it suffices to consider only boundary perturbations $\mathbf{Z} \in C^{2}\left(\Sigma_{T}\right)$.

REMARK 3.5. Since the domain $Q_{T}$ depends on the mapping $\boldsymbol{\kappa}$, we can also write $\nabla J\left(Q_{T}\right)[\mathbf{Z}]=\nabla J(\boldsymbol{\kappa})[\mathbf{Z}]$. Here, $\nabla J(\boldsymbol{\kappa})$ is obviously linear in $\mathbf{Z} \in \mathcal{Z}_{\text {ad }}$ and one can verify that it is bounded. Thus, $J$ is Gâteaux differentiable at $\boldsymbol{\kappa}$. In the same way, we can argue that $J$ is Gâteaux differentiable on an open neighbourhood $U$ of $\boldsymbol{\kappa}$. Moreover, one can prove that $\nabla J: U \rightarrow \mathcal{Z}_{\text {ad }}^{\prime}$ is continuous at $\boldsymbol{\kappa}$ by showing that $\|\nabla J(\boldsymbol{\kappa})-\nabla J(\tilde{\boldsymbol{\kappa}})\| \rightarrow 0$ as $\boldsymbol{\kappa} \rightarrow \tilde{\boldsymbol{\kappa}}$. This can be done by transforming the problem onto the reference domain $Q_{0}$, using the convergence of $\tilde{\boldsymbol{\kappa}}$ to $\boldsymbol{\kappa}$ in $C^{2}\left([0, T] \times \mathbb{R}^{d}\right)$ and the convergence in $H^{1,0}\left(Q_{0}\right)$ of the solutions $p \circ \tilde{\boldsymbol{\kappa}}$ to $p \circ \boldsymbol{\kappa}$ and $v \circ \tilde{\boldsymbol{\kappa}}$ to $v \circ \boldsymbol{\kappa}$ according to [18, Theorem 4.5 on pg. 166]. Hence, by using [15, pg. 41], we can conclude that $J$ is also Fréchet differentiable at $\boldsymbol{\kappa}$ and, therefore, the application of a gradient-based method for the numerical computations in Section 6 is justified (compare [14]).

4. Discretization of the shape optimization problem. In order to solve the shape optimization problem under consideration numerically, we need a suitable discretization of the sought domain. It can for example be represented by level sets or by a parametrization of its boundary, where it suffices to consider only the interior boundary as the exterior boundary is fixed. We employ here the latter approach since we will apply a boundary element method to compute the state and its adjoint. By restriction to two spatial dimensions and $C^{2}$-smooth star-shaped voids, we can employ a parametrization in space which is based on a Fourier series for an unknown radial function, having time dependent coefficients. Especially, we consider only boundary perturbation fields $\mathbf{Z} \in C^{2}\left(\Sigma_{T}\right)$, compare Remark 3.4.

Our choice of parametrization of the interior moving boundary $\Sigma_{T}$ of $Q_{T}$ is

$$
\Sigma_{T}=\left\{\left[\begin{array}{c}
t \\
\gamma(t, \theta)
\end{array}\right] \in \mathbb{R}^{3}: t \in[0, T], \theta \in[0,2 \pi)\right\},
$$

where the time-dependent parametrization $\gamma(t, \cdot):[0,2 \pi) \rightarrow \Gamma_{t}$ employs polar coordinates

$$
\gamma(t, \theta)=w(t, \theta)\left[\begin{array}{l}
\cos (\theta) \\
\sin (\theta)
\end{array}\right] .
$$

Here, $w(t, \theta)$ denotes the time- and angle-dependent radius, given by

$$
w(t, \theta):=\sum_{\ell=0}^{N_{L}} L_{\ell}(t)\left(\alpha_{0, \ell}+\sum_{k=1}^{N_{K}-1}\left\{\alpha_{k, \ell} \cos (k \theta)+\beta_{k, \ell} \sin (k \theta)\right\}+\alpha_{N_{K}, \ell} \cos \left(N_{K} \theta\right)\right),
$$

with $L_{\ell}(t)$ being appropriate dilations and translations of the Legendre polynomials of degree $\ell$.

Finding the optimal tube now corresponds to determining the unknown coefficients $\alpha_{k, \ell}$ and $\beta_{k, \ell}$ of the parametrization. Hence, we have the following finite dimensional problem:

$$
\text { Seek } \gamma^{\star} \in Z_{N} \text { such that } \nabla J\left(\gamma^{\star}\right)[\mathbf{Z}]=0 \text { for all } \mathbf{Z} \in Z_{N} \text {. }
$$


Here, $Z_{N}$ is the finite dimensional ansatz space of parametrizations. To compute the discrete shape gradient, we hence have to consider the directions

$$
(\mathbf{Z} \circ \gamma)(t, \theta)=L_{\ell}(t) \cos (k \theta)\left[\begin{array}{l}
\cos (\theta) \\
\sin (\theta)
\end{array}\right]
$$

for all $\ell=0, \ldots, N_{L}$ and $k=0, \ldots, N_{K}$, and

$$
(\mathbf{Z} \circ \gamma)(t, \theta)=L_{\ell}(t) \sin (k \theta)\left[\begin{array}{l}
\cos (\theta) \\
\sin (\theta)
\end{array}\right]
$$

for all $\ell=0, \ldots, N_{L}$ and $k=1, \ldots, N_{K}-1$.

With the specific parametrization at hand, the discrete shape gradient with respect to the parameters $t$ and $\theta$ reads

$\nabla J\left(Q_{T}\right)[\mathbf{Z} \circ \boldsymbol{\gamma}]=\int_{0}^{T} \int_{0}^{2 \pi}\left(\frac{\partial p}{\partial \mathbf{n}} \circ \boldsymbol{\gamma}\right)\left(\frac{\partial v}{\partial \mathbf{n}} \circ \boldsymbol{\gamma}\right)\left[\begin{array}{c}L_{1}(t) \\ \vdots \\ L_{N_{L}}(t)\end{array}\right] \otimes\left[\begin{array}{c}\sin \left(\left(N_{K}-1\right) \theta\right) \\ \vdots \\ \sin (\theta) \\ 1 \\ \cos (\theta) \\ \vdots \\ \cos \left(N_{K} \theta\right)\end{array}\right] w(t, \theta) \mathrm{d} \theta \mathrm{d} t$, compare (3.5), where we plugged in the choices for the perturbation fields (4.2) and (4.3), respectively, and used the parametrization $\gamma$ to compute the normal $\mathbf{n}$.

The integral in the shape gradient (3.5) is computed by using a trapezoidal rule in space and a trapezoidal rule with a singularity correction at the endpoint $t=T$ in time (see the next section for details). The Legendre polynomials are computed by using their three term recurrence formula as described in [25], and are normalized afterwards while the Fourier series is evaluated efficiently by the fast Fourier transform.

The gradient-based method of our choice is the quasi-Newton method, updated by the inverse BFGS rule without damping, cf. [10]. A second order line search is applied to find an appropriate step size in the quasi-Newton method. For an overview of possible other optimization algorithms in general, see [6, 9].

5. Solving parabolic boundary value problems. We shall describe the numerical method for solving the state and adjoint equation by using a boundary integral formulation. Since this is the approach that was already taken in [13] for a fixed boundary, we focus in this section on the changes for the time dependent-case.

Both, the state and the adjoint equation, are Dirichlet problems of the heat equation with homogeneous initial conditions. In the case of the adjoint equation this becomes apparent after the change of variables $t \mapsto T-t$.

The boundary integral approach has distinct advantages over domain based approaches, because it is not necessary to mesh a time-dependent domain or consider the transported problem in a cylindrical domain. Instead, we solve the Green's integral equation. For a time-dependent boundary, it has the form

$$
\frac{1}{2} \phi(t, \mathbf{x})=\mathcal{V} \gamma_{1}^{-} \phi(t, \mathbf{x})-\mathcal{K} \phi(t, \mathbf{x}), \quad(t, \mathbf{x}) \in \Sigma_{T} \cup \Sigma^{f} .
$$

Here, $\mathcal{V}$ and $\mathcal{K}$ are the thermal single and double layer operators defined below, and $\phi$ is a solution to the source-free heat equation with homogeneous initial conditions. 
Because of the time dependence of the spatial surface $\Gamma_{t}$ the normal trace operator is

$$
\gamma_{1}^{ \pm} \phi:= \begin{cases}\frac{\partial \phi}{\partial \mathbf{n}} \mp \frac{1}{2}\langle\mathbf{V}, \mathbf{n}\rangle \phi & \text { on } \Gamma_{t}, \\ \frac{\partial \phi}{\partial \mathbf{n}} & \text { on } \Gamma^{f},\end{cases}
$$

where $\langle\mathbf{V}, \mathbf{n}\rangle$ is the normal velocity, which can be computed analytically from the parametrization $\gamma$ given in (4.1) as $\mathbf{V}(t, \theta)=\frac{\mathrm{d}}{\mathrm{d} t} \gamma(t, \theta)$. The extra term in the definition of $\gamma_{1}^{ \pm}$arises from the Reynolds transport theorem in the derivation of (5.1). Details can be found in [30].

For the discretization of (5.1), it is desirable to have a method that can be easily adapted to time-dependent geometries, hence we use the Nyström discretization method of $[29,30]$. To that end, we write the thermal layer potentials in the form

$$
\begin{aligned}
& \mathcal{V} \phi(t, \mathbf{x})=\frac{1}{\sqrt{4 \pi}} \int_{0}^{t} \frac{1}{\sqrt{t-\tau}} V \phi(t, \tau, \mathbf{x}) \mathrm{d} \tau \\
& \mathcal{K} \phi(t, \mathbf{x})=\frac{1}{\sqrt{4 \pi}} \int_{0}^{t} \frac{1}{\sqrt{t-\tau}} K \phi(t, \tau, \mathbf{x}) \mathrm{d} \tau
\end{aligned}
$$

where

$$
\begin{aligned}
& V \phi(t, \tau, \mathbf{x})=\int_{\Gamma_{\tau} \cup \Gamma_{f}^{f}} \frac{1}{(4 \pi(t-\tau))^{\frac{d-1}{2}}} \exp \left(-\frac{\|\mathbf{x}-\mathbf{y}\|^{2}}{4(t-\tau)}\right) \phi(\mathbf{y}, \tau) \mathrm{d} \sigma_{\mathbf{y}} \\
& K \phi(t, \tau, \mathbf{x})=\int_{\Gamma_{\tau} \cup \Gamma^{f}} \frac{1}{(4 \pi(t-\tau))^{\frac{d-1}{2}}} \gamma_{1, \mathbf{y}}^{+}\left[\exp \left(-\frac{\|\mathbf{x}-\mathbf{y}\|^{2}}{4(t-\tau)}\right)\right] \phi(\mathbf{y}, \tau) \mathrm{d} \sigma_{\mathbf{y}},
\end{aligned}
$$

and $\Gamma_{\tau} \cup \Gamma^{f}=\partial \Omega_{\tau}$, i.e., the union of the free and the fixed boundary. Here, $\gamma_{1, \mathbf{y}}^{+}$is the normal trace (5.2) evaluated at $(\mathbf{y}, \tau)$.

The kernel in the above time-dependent surface potentials is the Green's function of the $(d-1)$-dimensional heat equation. Thus, they may be regarded as PoissonWeierstrass integrals defined on a surface instead of the usual plane. As in the planar case, these integrals are smooth functions in all variables when $0 \leq \tau \leq t$. The limiting behavior of these functions as $\tau \rightarrow t$ is

$$
\begin{aligned}
V \phi(t, \tau, \mathbf{x}) & =\phi(t, \tau, \mathbf{x})+\mathcal{O}(t-\tau), \\
K \phi(t, \tau, \mathbf{x}) & =H(t, \mathbf{x}) \phi(\mathbf{x})+\mathcal{O}(t-\tau),
\end{aligned}
$$

where $H(\cdot)$ is the mean curvature of the surface $\Gamma_{t} \cup \Gamma^{f}$, see [30].

Since the functions $V \phi$ and $K \phi$ are smooth, the integral operators in (5.3) and (5.4) have a $(t-\tau)^{-1 / 2}$ singularity, which suggests to use the trapezoidal rule with a singularity correction at the endpoint $t=\tau$. It is shown in [29] that the rule

$$
\mathcal{V} \phi\left(\mathbf{x}, t_{n}\right)=\frac{h}{\sqrt{4 \pi}} \sum_{j=0}^{n-1} \frac{1}{\sqrt{t_{n}-t_{j}}} V\left(t_{n}, t_{j}\right) \phi\left(\mathbf{x}, t_{j}\right)+\mu_{n} \psi\left(\mathbf{x}, t_{n}\right)+\epsilon_{h},
$$

where $h$ is the time step length, $t_{j}=h j$ and

$$
\mu_{n}=\sqrt{\frac{t_{n}}{\pi}}-\frac{h}{\sqrt{4 \pi}} \sum_{j=0}^{n-1} \frac{1}{\sqrt{t_{n}-t_{j}}},
$$


has a quadrature error of $\epsilon_{h}=\mathcal{O}\left(h^{3 / 2}\right)$. Here, the prime at the summation sign indicates that the $j=0$ term in the sum is multiplied by the factor $1 / 2$. For the double layer analogous result holds when the $\mu_{n}$-term is multiplied by the curvature. A fully discrete version is obtained by approximating the surface integrals in (5.5) and (5.6) by a surface quadrature rule, usually a composite rule that integrates polynomials on triangular patches exactly. If the spatial mesh width $h_{s}$ satisfies $\sqrt{h_{s}} \sim h$ and the spatial rule has at least degree of precision two then the quadrature error in (5.8) can be preserved, see [29]. In the time dependent case, these rules are constructed on $\Gamma_{0} \cup \Gamma^{f}$ and then mapped to $\Gamma_{t} \cup \Gamma^{f}$.

For the state equation, the solution is smooth and the Nyström method based on the above quadrature is used to compute the normal trace of the solution. Thus the Neumann data at the quadrature nodes is computed from (5.1) by substituting the given Dirichlet data of (2.4). This gives approximate values of the shape functional (2.9) and the boundary condition in the adjoint state (3.6).

The next task is to compute the Neumann data in the shape gradient (3.5) by solving the adjoint state. As already observed in [13], the adjoint equation (after time transformation $t \mapsto T-t$ ) has a singularity at $\tau=0$ because the homogeneous initial condition is not compatible with the in general non vanishing Dirichlet condition at $t=0$.

It can be concluded from (5.7) and Green's integral equation that the Neumann data has a $t^{-1 / 2}$-singularity at $t=0$. To preserve the $\mathcal{O}\left(h^{\frac{3}{2}}\right)$ accuracy, the time quadrature rule (5.8) must be modified with singularity corrections on both endpoints. Since the normal velocity of the boundary does not appear in (5.7), the derivation and the weights of this rule are identical to the case of a steady boundary. Since this can be found in [13], it is not repeated here.

6. Numerical experiments. We shall present some numerical results in order to illustrate the approach. To this end, the exterior, fixed boundary of the spacetime domain is chosen as the mantle of the cylinder with radius 1 , where its height corresponds to the time interval $(0, T)=(0,1)$. We choose $N_{t}=90$ time intervals and, for every time step, $N_{\mathbf{x}}=80$ spatial points. The void is depicted in Figure 6.1. It has an explicit representation and is discretized by the same number of time intervals and spatial points as the exterior boundary.

We first solve the forward problem to construct the desired Neumann data $g$. We hence consider the desired shape found in Figure 6.1 and choose the Dirichlet data $f(t, \cdot)=t$, which matches with the initial data $u(0, \cdot)=0$ in $\Omega_{0}$. In order to avoid an inverse crime, we use an indirect boundary element approach by solving the thermal single layer equation and then recover the Neumann data by applying the thermal adjoint operator. In addition, we add $1 \%$ random noise to the synthetic data.

Now, we can tackle the inverse problem. For the parametrization of the interior boundary, we choose 16 Fourier coefficients in space $\left(N_{K}=8\right)$ and 10 Legendre polynomials in time $\left(N_{L}=9\right)$, leading to 160 design parameters in total. As an initial guess for the free inner boundary, we choose the cylinder of radius 0.3 . We perform 100 iterations in the optimization procedure and use a quasi-Newton method updated by the limited memory inverse BFGS rule, where 10 updates are stored, see [24] for example.

In Figure 6.2 on the right, the evolution of the shape gradient during the course of the minimization algorithm is shown, while on the left the evolution of the functional is displayed. In Figure 6.3, we can see the $\ell^{2}$-error in the shape coefficients corresponding to the shape error. We clearly observe convergence of the minimization algorithm. 


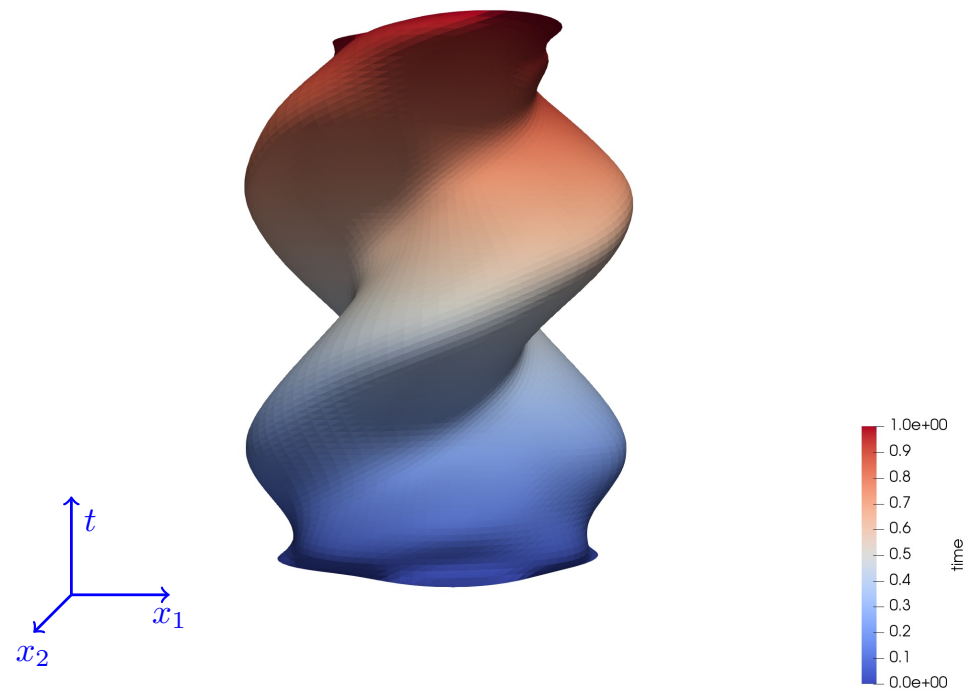

Figure 6.1: First example: given inclusion in space and time.
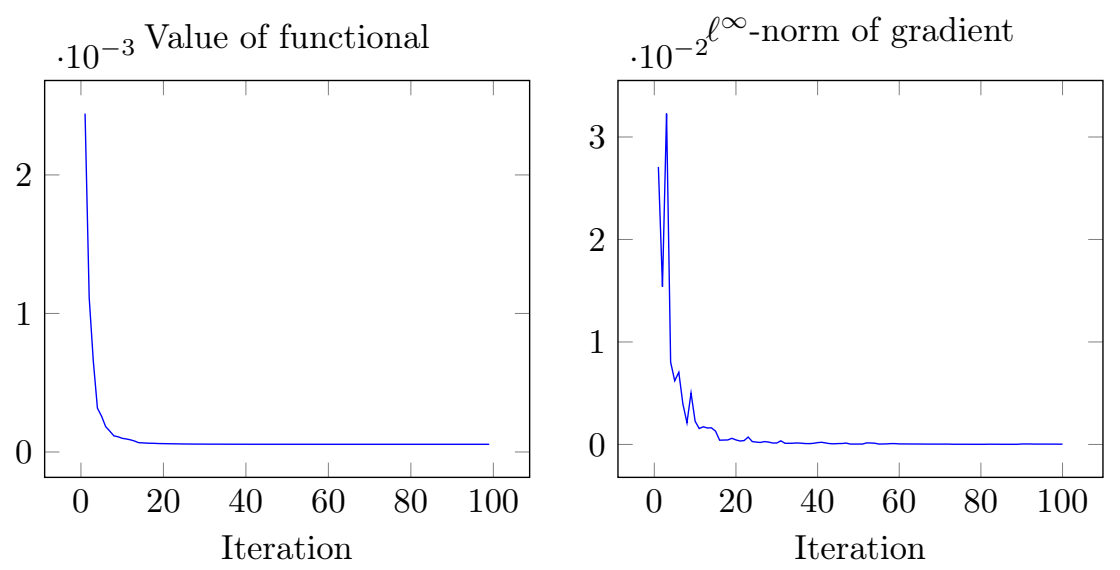

Figure 6.2

First example: the histories of the functional (left) and of the shape gradient (right).

In Figure 6.4, we present the final reconstruction of the space-time shape, where the wireframe corresponds to the exact shape and the solid shape is its reconstruction. When looking at the time slices, one can observe that the spatial boundary $\Gamma_{t}$ is very well reconstructed for the intermediate time slices with $0<t<T$. Whereas, the reconstruction is not very good at the starting time $t=0$ and the stopping time $T=1$. Here, we have no measurement data either of the future or of the past which enter the shape functional (2.9). This makes the shape reconstruction more ill-posed in comparison to intermediate time slices. 


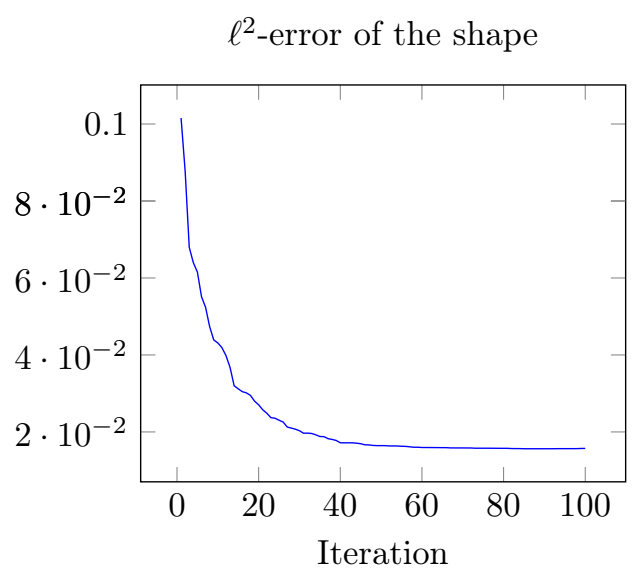

Figure 6.3: $\ell^{2}$-error of the shape coefficients corresponding to the difference in the shapes.

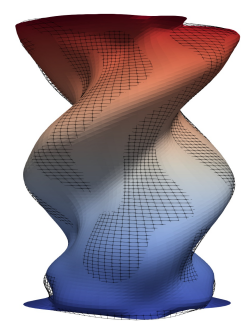

(a) View with the $x$-axis in front.

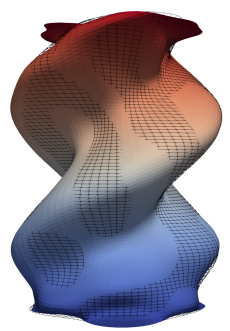

(b) View with the $x_{2}$-axis in front.

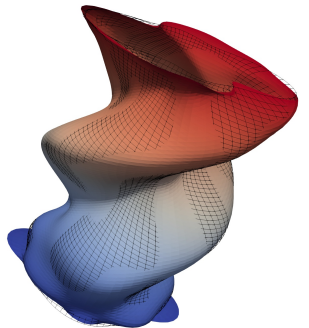

(c) Three-dimensional view.

Figure 6.4: First example: The desired shape as a wireframe together with the reconstructed shape in solid. The time corresponds to the $z$-axis.

To show the feasibility of our numerical computations, we reconstructed a second, nonsymmetric inclusion (compare Figure 6.5), using the same set-up and parameters as for the first inclusion.

In Figure 6.6, the final reconstruction is shown in solid and the desired shape in wireframe. As for the first numerical example, the reconstruction is fairly good for times away from the starting and ending time. Nonetheless, the reconstructed shape is a bit smoother compared to the desired shape. The value of the functional, the $\ell^{\infty}$-norm of the gradient, and the $\ell^{2}$-error of the shape are not depicted since they evolve in a similar manner as in the first example shown in Figures 6.2 and 6.3.

7. Conclusion. In this article, we solved a time-dependent shape reconstruction problem by means of shape optimization. We computed the shape derivative of the tracking-type functional for the Neumann data with the help of the perturbation of identity. It turned out that this shape derivative coincides with the one when the 


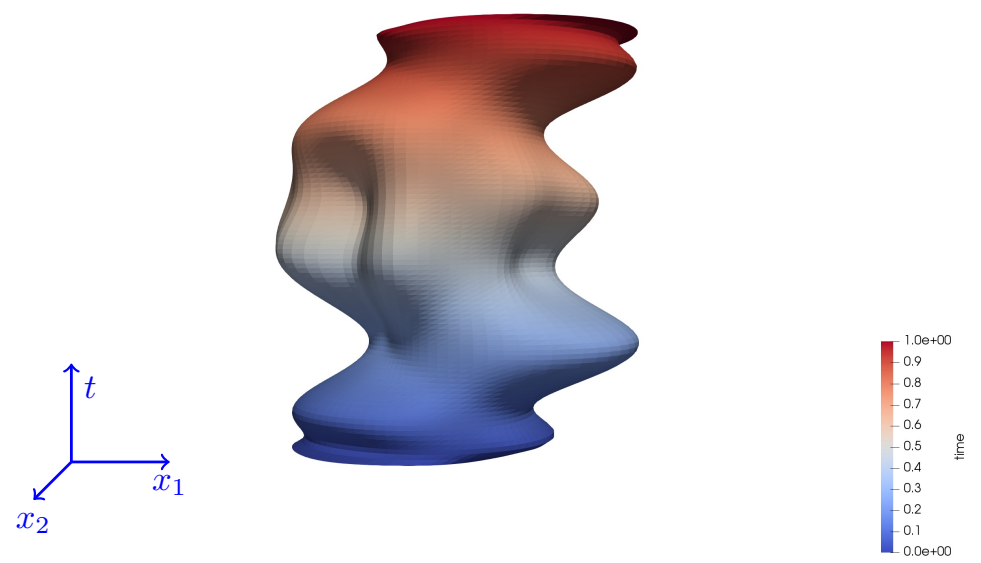

Figure 6.5: Second example: given inclusion in space and time.

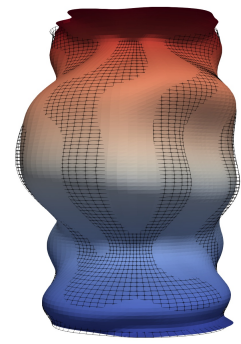

(a) View with the $x$-axis in front.

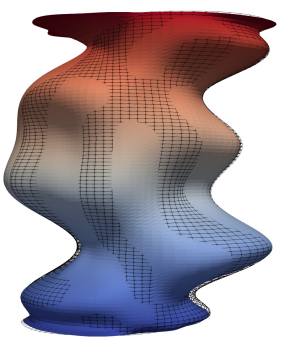

(b) View with the $x_{2}$-axis in front.

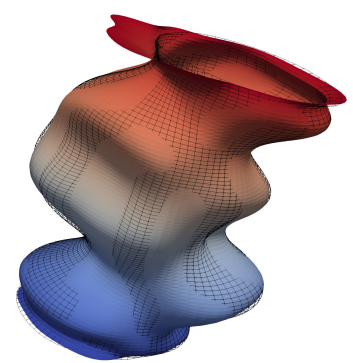

(c) Three-dimensional view.

Figure 6.6: Second example: The desired shape as a wireframe together with the reconstructed shape in solid. The time corresponds to the $z$-axis.

void is time-independent. We also demonstrated by numerical experiments that it is indeed possible to reconstruct a time-dependent shape by the proposed approach. By restricting to star-shaped voids, we have been able to compute the error between the desired shape and the reconstructed shape. The convergence of the minimization algorithm has clearly been observed.

Appendix A. Local shape derivative. The proof of the local shape derivative follows the lines of [2]. We state here the adjustment to the time-dependent setting.

We first present two general lemmas, which are used later. We consider a mapping $\boldsymbol{\xi}$, which maps a domain $\Omega_{\tau}$ to a domain $\Omega_{\varsigma}$ and satisfies a uniformity condition as in (2.2). We will use the lemmas for $\boldsymbol{\xi}=\boldsymbol{\kappa}$ and $\boldsymbol{\xi}=\mathbf{I}+s \mathbf{Z}$. Let us denote $Q_{\tau}=$ $\cup_{\tau}\left(\{\tau\} \times \Omega_{\tau}\right)$ and analogously for $Q_{\varsigma}$ and the lateral area by $\Sigma_{\tau}$ or $\Sigma_{\varsigma}$, respectively. 
Lemma A.1. For $v$ smooth enough it holds

$$
(\nabla v) \circ \boldsymbol{\xi}=(\mathrm{D} \boldsymbol{\xi})^{-\top} \nabla(v \circ \boldsymbol{\xi})
$$

and

$$
\left(\partial_{t} v\right) \circ \boldsymbol{\xi}=\partial_{t}(v \circ \boldsymbol{\xi})-(\mathrm{D} \boldsymbol{\xi})^{-\top} \nabla(v \circ \boldsymbol{\xi}) \cdot \partial_{t} \boldsymbol{\xi} .
$$

Proof. By the chain rule, we can compute

$$
\nabla(v \circ \boldsymbol{\xi})=(\mathrm{D} \boldsymbol{\xi})^{\top}(\nabla v) \circ \boldsymbol{\xi}
$$

from where (A.1) follows immediately. Moreover, the multivariable chain rule yields

$$
\partial_{t}(v \circ \boldsymbol{\xi})=\left(\partial_{t} v\right) \circ \boldsymbol{\xi}+(\nabla v) \circ \boldsymbol{\xi} \cdot \partial_{t} \boldsymbol{\xi}
$$

since only the spatial component is affected by the composition with $\boldsymbol{\xi}$. By using (A.1), we get (A.2).

Notice that the identities (A.1) and (A.2) are also stated in [23, pg. 43] in the setting of the speed method.

LemmA A.2. Let $v \in \hat{H}^{1, \frac{1}{2}}\left(Q_{\varsigma}\right)$ and $\varphi \in \tilde{H}_{0}^{1, \frac{1}{2}}\left(Q_{\varsigma}\right)$. Then, the transport of

$$
S(v, \varphi):=\int_{0}^{T} \int_{\Omega_{\varsigma}}\left\{\nabla v \cdot \nabla \varphi+\partial_{t} v \varphi\right\} \mathrm{d} \mathbf{x} \mathrm{d} t=\int_{0}^{T} \int_{\Omega_{\varsigma}} q \varphi \mathrm{d} \mathbf{x} \mathrm{d} t
$$

from $Q_{\varsigma}$ to $Q_{\tau}$ gives the parabolic problem

$$
\int_{0}^{T} \int_{\Omega_{\tau}} \partial_{t} v^{\tau, \varsigma} \varphi^{\tau, \varsigma} \mathrm{d} \mathbf{x} \mathrm{d} t+\int_{0}^{T} a\left(t ; v^{\tau, \varsigma}, \varphi^{\tau, \varsigma}\right) \mathrm{d} t=\int_{0}^{T} \int_{\Omega_{\tau}} q^{t} \varphi^{\tau, \varsigma} \mathrm{d} \mathbf{x} \mathrm{d} t
$$

with

$$
\begin{aligned}
a\left(t ; v^{\tau, \varsigma}, \varphi^{\tau, \varsigma}\right):= & \int_{\Omega_{\tau}}\left\langle(\mathrm{D} \boldsymbol{\xi})^{-\mathrm{\top}} \nabla v^{\tau, \varsigma},(\mathrm{D} \boldsymbol{\xi})^{-\mathrm{\top}} \nabla \varphi^{\tau, \varsigma}\right\rangle \mathrm{d} \mathbf{x} \\
& -\int_{\Omega_{\tau}}\left\langle(\mathrm{D} \boldsymbol{\xi})^{-\mathrm{\top}} \nabla v^{\tau, \varsigma}, \partial_{t} \boldsymbol{\xi} \varphi^{t}\right\rangle \mathrm{d} \mathbf{x} \\
& -\int_{\Omega_{\tau}}\left\langle(\mathrm{D} \boldsymbol{\xi})^{-\top} \frac{1}{\operatorname{det}(\mathrm{D} \boldsymbol{\xi})} \nabla(\operatorname{det}(\mathrm{D} \boldsymbol{\xi})) \varphi^{\tau, \varsigma},(\mathrm{D} \boldsymbol{\xi})^{-\top} \nabla v^{\tau, \varsigma}\right\rangle \mathrm{d} \mathbf{x},
\end{aligned}
$$

where $v^{\tau, \varsigma}=v \circ \boldsymbol{\xi}$ and similarly for $\varphi^{\tau, \varsigma}$ and $q^{\tau, \varsigma}$.

Proof. With the aid of Lemma A.1, the transport of (A.3) from $Q_{\varsigma}$ onto $Q_{\tau}$ gives

$$
\begin{aligned}
& \int_{0}^{T} \int_{\Omega_{\tau}} \operatorname{det}(\mathrm{D} \boldsymbol{\xi})(\mathrm{D} \boldsymbol{\xi})^{-\mathrm{T}} \nabla(v \circ \boldsymbol{\xi}) \cdot(\mathrm{D} \boldsymbol{\xi})^{-\mathrm{T}} \nabla(\varphi \circ \boldsymbol{\xi}) \mathrm{d} \mathbf{x} \mathrm{d} t \\
& \quad+\int_{0}^{T} \int_{\Omega_{\tau}} \operatorname{det}(\mathrm{D} \boldsymbol{\xi})\left[\partial_{t}(\varphi \circ \boldsymbol{\xi})(v \circ \boldsymbol{\xi})-(\mathrm{D} \boldsymbol{\xi})^{-\mathrm{T}} \nabla(v \circ \boldsymbol{\xi}) \cdot \partial_{t} \boldsymbol{\xi}(\varphi \circ \boldsymbol{\xi})\right] \mathrm{d} \mathbf{x} \mathrm{d} t \\
& \quad=\int_{0}^{T} \int_{\Omega_{\tau}} \operatorname{det}(\mathrm{D} \boldsymbol{\xi})(q \circ \boldsymbol{\xi})(\varphi \circ \boldsymbol{\xi}) \mathrm{d} \mathbf{x} \mathrm{d} t .
\end{aligned}
$$


Using Green's first identity and the zero boundary condition yields

$$
\begin{aligned}
& \int_{0}^{T} \int_{\Omega_{\tau}}-\operatorname{div}\left(\operatorname{det}(\mathrm{D} \boldsymbol{\xi})(\mathrm{D} \boldsymbol{\xi})^{-1}(\mathrm{D} \boldsymbol{\xi})^{-\mathrm{T}} \nabla(v \circ \boldsymbol{\xi})\right)(\varphi \circ \boldsymbol{\xi}) \mathrm{d} \mathbf{x} \mathrm{d} t \\
& \quad+\int_{0}^{T} \int_{\Omega_{\tau}} \operatorname{det}(\mathrm{D} \boldsymbol{\xi})\left[\partial_{t}(v \circ \boldsymbol{\xi})-(\mathrm{D} \boldsymbol{\xi})^{-\top} \nabla(v \circ \boldsymbol{\xi}) \cdot \partial_{t} \boldsymbol{\xi}\right](\varphi \circ \boldsymbol{\xi}) \mathrm{d} \mathbf{x} \mathrm{d} t \\
& \quad=\int_{0}^{T} \int_{\Omega_{\tau}} \operatorname{det}(\mathrm{D} \boldsymbol{\xi})(q \circ \boldsymbol{\xi})(\varphi \circ \boldsymbol{\xi}) \mathrm{d} \mathbf{x} \mathrm{d} t .
\end{aligned}
$$

Thus, in the strong formulation, we have when dividing by $\operatorname{det}(\mathrm{D} \boldsymbol{\xi})$ that

$$
\begin{aligned}
-\frac{1}{\operatorname{det}(\mathrm{D} \boldsymbol{\xi})} \operatorname{div}\left(\operatorname{det}(\mathrm{D} \boldsymbol{\xi})(\mathrm{D} \boldsymbol{\xi})^{-1}(\mathrm{D} \boldsymbol{\xi})^{-\mathrm{T}} \nabla(v \circ \boldsymbol{\xi})\right) \\
+\partial_{t}(v \circ \boldsymbol{\xi})-(\mathrm{D} \boldsymbol{\xi})^{-\mathrm{T}} \nabla(v \circ \boldsymbol{\xi}) \cdot \partial_{t} \boldsymbol{\xi}=q \circ \boldsymbol{\xi} \text { in } Q_{\tau} .
\end{aligned}
$$

Rewriting gives

$$
\begin{aligned}
-\operatorname{div}\left((\mathrm{D} \boldsymbol{\xi})^{-1}(\mathrm{D} \boldsymbol{\xi})^{-\mathrm{T}} \nabla v^{\tau, \varsigma}\right)+\partial_{t} v^{\tau, \varsigma}-(\mathrm{D} \boldsymbol{\xi})^{-\mathrm{T}} \nabla v^{\tau, \varsigma} \cdot \partial_{t} \boldsymbol{\xi} & \\
& -\frac{1}{\operatorname{det}(\mathrm{D} \boldsymbol{\xi})} \nabla(\operatorname{det}(\mathrm{D} \boldsymbol{\xi})) \cdot(\mathrm{D} \boldsymbol{\xi})^{-1}(\mathrm{D} \boldsymbol{\xi})^{-\mathrm{T}} \nabla v^{\tau, \varsigma}=q^{\tau, \varsigma} \text { in } Q_{\tau}
\end{aligned}
$$

Testing again with a function $\varphi^{\tau, \varsigma}$ gives the weak formulation

$$
\begin{aligned}
& \int_{0}^{T} \int_{\Omega_{\tau}}-\operatorname{div}\left((\mathrm{D} \boldsymbol{\xi})^{-1}(\mathrm{D} \boldsymbol{\xi})^{-\mathrm{T}} \nabla v^{\tau, \varsigma}\right) \varphi^{\tau, \varsigma} \mathrm{d} \mathbf{x} \mathrm{d} t+\int_{0}^{T} \int_{\Omega_{\tau}} \partial_{t} v^{\tau, \varsigma} \varphi^{\tau, \varsigma} \mathrm{d} \mathbf{x} \mathrm{d} t \\
& \quad-\int_{0}^{T} \int_{\Omega_{\tau}}(\mathrm{D} \boldsymbol{\xi})^{-\top} \nabla v^{\tau, \varsigma} \cdot \partial_{t} \boldsymbol{\xi} \varphi^{\tau, \varsigma} \mathrm{d} \mathbf{x} \mathrm{d} t \\
& -\int_{0}^{T} \int_{\Omega_{\tau}} \frac{1}{\operatorname{det}(\mathrm{D} \boldsymbol{\xi})} \nabla(\operatorname{det}(\mathrm{D} \boldsymbol{\xi})) \cdot(\mathrm{D} \boldsymbol{\xi})^{-1}(\mathrm{D} \boldsymbol{\xi})^{-\top} \nabla v^{\tau, \varsigma} \varphi^{\tau, \varsigma} \mathrm{d} \mathbf{x} \mathrm{d} t \\
& \quad=\int_{0}^{T} \int_{\Omega_{\tau}} q^{\tau, \varsigma} \varphi^{\tau, \varsigma} \mathrm{d} \mathbf{x} \mathrm{d} t,
\end{aligned}
$$

which can be reformulated by using the divergence theorem with vanishing boundary terms to

$$
\begin{aligned}
\int_{0}^{T} & \int_{\Omega_{\tau}}(\mathrm{D} \boldsymbol{\xi})^{-\mathrm{T}} \nabla v^{\tau, \varsigma} \cdot(\mathrm{D} \boldsymbol{\xi})^{-\mathrm{T}} \nabla \varphi^{\tau, \varsigma} \mathrm{d} \mathbf{x} \mathrm{d} t+\int_{0}^{T} \int_{\Omega_{\tau}} \partial_{t} v^{\tau, \varsigma} \varphi^{\tau, \varsigma} \mathrm{d} \mathbf{x} \mathrm{d} t \\
- & \int_{0}^{T} \int_{\Omega_{\tau}}\left\langle(\mathrm{D} \boldsymbol{\xi})^{-\top} \nabla v^{\tau, \varsigma}, \partial_{t} \boldsymbol{\xi} \varphi^{t}\right\rangle \mathrm{d} \mathbf{x} \mathrm{d} t \\
- & \int_{0}^{T} \int_{\Omega_{\tau}}\left\langle(\mathrm{D} \boldsymbol{\xi})^{-\top} \frac{1}{\operatorname{det}(\mathrm{D} \boldsymbol{\xi})} \nabla(\operatorname{det}(\mathrm{D} \boldsymbol{\xi})) \varphi^{\tau, \varsigma},(\mathrm{D} \boldsymbol{\xi})^{-\boldsymbol{\top}} \nabla v^{\tau, \varsigma}\right\rangle \mathrm{d} \mathbf{x} \mathrm{d} t \\
& =\int_{0}^{T} \int_{\Omega_{\tau}} q^{\tau, \varsigma} \varphi^{\tau, \varsigma} \mathrm{d} \mathbf{x} \mathrm{d} t .
\end{aligned}
$$

From here, the claim follows immediately.

In order to compute the local shape derivative, we first introduce the material derivative to (2.4) as stated in the following lemma. 
Lemma A.3. Let us set $v^{t, s}:=v_{t, s} \circ(\mathbf{I}+s \mathbf{Z})$ for $\mathbf{Z} \in \mathcal{Z}_{\text {ad }}$, where $v_{t, s}$ is defined in Section 3.2. The material derivative of (2.4), which is defined as the limit (see also $(3.2))$

$$
\dot{v}:=\lim _{s \rightarrow 0} \frac{v^{t, s}-v}{s},
$$

exists in $\hat{H}_{0}^{1, \frac{1}{2}}\left(Q_{T}\right)$ and satisfies

$$
S(\dot{v}, \varphi)=G(\varphi) \text { for all } \varphi \in \tilde{H}_{0}^{1, \frac{1}{2}}\left(Q_{T}\right),
$$

where $S$ is given by (2.5) and

$$
G(\varphi)=\int_{0}^{T} \int_{\Omega_{t}}\left\{\left(\mathrm{D} \mathbf{Z}+\mathrm{D} \mathbf{Z}^{\boldsymbol{\top}}\right) \nabla v \cdot \nabla \varphi+\varphi \nabla(\operatorname{div} \mathbf{Z}) \cdot \nabla v+\left(\partial_{t} \mathbf{Z}\right) \cdot \nabla v \varphi\right\} \mathrm{d} \mathbf{x} \mathrm{d} t
$$

Proof. As an immediate consequence of [18, Chapter IV, Theorem 9.1], the solution $v_{t, s}$ lies in $\hat{H}^{2,1}\left(Q_{T}^{s}\right)$ under our smoothness assumptions. Notice that the increased regularity of the solution of the differential equation is needed for the boundary condition of the local shape derivative (3.4) and also in the adjoint problem (3.6)).

We have for the perturbed bilinear form

$$
S_{s}\left(v_{t, s}, \varphi\right):=\int_{0}^{T} \int_{\Omega_{t, s}}\left\{\partial_{t} v_{t, s} \varphi+\nabla v_{t, s} \cdot \nabla \varphi\right\} \mathrm{d} \mathbf{x} \mathrm{d} t
$$

that $S_{s}\left(v_{t, s}, \varphi\right)=0$ for all $\varphi \in \tilde{H}_{0}^{1, \frac{1}{2}}\left(Q_{T}^{s}\right)$. The existence and uniqueness of a solution follows as in Theorem 2.2 by using that the transformation $\boldsymbol{\kappa}+s \mathbf{Z} \circ \boldsymbol{\kappa}$ satisfies again a uniformity condition as stated in (2.2). With similar computations as in the proof of Lemma A.2, when setting $\boldsymbol{\xi}=\mathbf{I}+s \mathbf{Z}, \Omega_{\tau}=\Omega_{t}$ and $\Omega_{\varsigma}=\Omega_{t, s}$, the transformation of the integral in (A.9) back onto $\Omega_{t}$ reads

$$
\begin{aligned}
S_{s}\left(v_{t, s}, \varphi\right)=\int_{0}^{T} \int_{\Omega_{t}} \operatorname{det}(\mathrm{D}(\mathbf{I} & +s \mathbf{Z}))\left[\left\{\partial_{t} v^{t, s}-(\mathrm{D}(\mathbf{I}+s \mathbf{Z}))^{-\boldsymbol{\top}} \nabla v^{t, s} \cdot \partial_{t}(\mathbf{I}+s \mathbf{Z})\right\} \varphi^{s}\right. \\
+ & \left.(\mathrm{D}(\mathbf{I}+s \mathbf{Z}))^{-\boldsymbol{\top}} \nabla v^{t, s} \cdot(\mathrm{D}(\mathbf{I}+s \mathbf{Z}))^{-\mathbf{T}} \nabla \varphi^{s}\right] \mathrm{d} \mathbf{x} \mathrm{d} t
\end{aligned}
$$

where we have set $v^{t, s}:=v_{t, s} \circ(\mathbf{I}+s \mathbf{Z})$ and $\varphi^{s}$ analogously. We define this bilinear form on the unperturbed domain as

$$
\begin{aligned}
S^{s}(w, \varphi) & :=\int_{0}^{T} \int_{\Omega_{t}} \operatorname{det}(\mathrm{D}(\mathbf{I}+s \mathbf{Z})) \\
& {\left[\mathbf{B}^{s} \nabla w \cdot \nabla \varphi+\partial_{t} w \varphi-(\mathrm{D}(\mathbf{I}+s \mathbf{Z}))^{-\mathbf{T}} \nabla w \cdot \partial_{t}(\mathbf{I}+s \mathbf{Z}) \varphi\right] \mathrm{d} \mathbf{x} \mathrm{d} t, }
\end{aligned}
$$

where

$$
\mathbf{B}^{s}=(\mathrm{D}(\mathbf{I}+s \mathbf{Z}))^{-1}(\mathrm{D}(\mathbf{I}+s \mathbf{Z}))^{-\boldsymbol{\top}} .
$$

Note that the last term in the definition of $S^{S}(w, \varphi)$ is new in comparison with [2].

We conclude the following statement:

$$
S_{s}\left(v_{t, s}, \varphi\right)=0 \text { for all } \varphi \in \tilde{H}_{0}^{1, \frac{1}{2}}\left(Q_{T}^{s}\right)
$$

for $v_{t, s} \in \hat{H}^{2,1}\left(Q_{T}^{s}\right)$ is equivalent to

$$
S^{s}\left(v^{t, s}, \varphi\right)=0 \text { for all } \varphi \in \tilde{H}_{0}^{1, \frac{1}{2}}\left(Q_{T}\right)
$$


for $v^{t, s} \in \hat{H}^{2,1}\left(Q_{T}\right)$. Integrating by parts, where we use the zero boundary values of the test function, and dividing by $\operatorname{det}(\mathrm{D}(\mathbf{I}+s \mathbf{Z}))$ verifies that (A.10) is equivalent to the formulation

$$
\begin{aligned}
& \partial_{t} v^{t, s}-(\mathrm{D}(\mathbf{I}+s \mathbf{Z}))^{-\top} \nabla v^{t, s} \cdot \partial_{t}(\mathbf{I}+s \mathbf{Z}) \\
& -\frac{1}{\operatorname{det}(\mathrm{D}(\mathbf{I}+s \mathbf{Z}))} \nabla(\operatorname{det}(\mathrm{D}(\mathbf{I}+s \mathbf{Z}))) \cdot \mathbf{B}^{s} \nabla v^{t, s}-\operatorname{div}\left(\mathbf{B}^{s} \nabla v^{t, s}\right)=0 \\
& \text { in } \bigcup_{0<t<T}\left(\{t\} \times \Omega_{t}\right) \text {. }
\end{aligned}
$$

Because of $S(v, \varphi)=0$ and $S^{s}\left(v^{t, s}, \varphi\right)=0$, it holds

$$
S\left(v^{t, s}-v, \varphi\right)=-S^{s}\left(v^{t, s}, \varphi\right)+S\left(v^{t, s}, \varphi\right) .
$$

We can therefore consider

$$
\frac{1}{s} S\left(v^{t, s}-v, \varphi\right)=G_{s}(\varphi) \text { for all } \varphi \in \tilde{H}_{0}^{1, \frac{1}{2}}\left(Q_{T}\right)
$$

for the computation of the material derivative, where

$$
\begin{aligned}
G_{s}(\varphi) & =\frac{1}{s} \int_{0}^{T} \int_{\Omega_{t}}\left\{-\operatorname{det}(\mathrm{D}(\mathbf{I}+s \mathbf{Z})) \mathbf{B}^{s} \nabla v^{t, s} \cdot \nabla \varphi-\operatorname{det}(\mathrm{D}(\mathbf{I}+s \mathbf{Z})) \partial_{t} v^{t, s} \varphi\right. \\
& +\operatorname{det}(\mathrm{D}(\mathbf{I}+s \mathbf{Z}))(\mathrm{D}(\mathbf{I}+s \mathbf{Z}))^{-\top} \nabla v^{t, s} \cdot \partial_{t}(\mathbf{I}+s \mathbf{Z}) \varphi \\
& \left.+\partial_{t} v^{t, s} \varphi+\nabla v^{t, s} \cdot \nabla \varphi\right\} \mathrm{d} \mathbf{x} \mathrm{d} t
\end{aligned}
$$

Herein, the second line is new in comparison with [2].

We reformulate the expression for $G_{s}(\varphi)$ the same way as in [2] and we arrive at

$$
\begin{aligned}
G_{s}(\varphi)=\frac{1}{s} \int_{0}^{T} \int_{\Omega_{t}}\left\{\left[\mathbf{I}-\mathbf{B}^{s}\right] \nabla v^{t, s} \cdot \nabla \varphi\right. & \\
& \left.+\frac{\varphi}{\operatorname{det}(\mathrm{D}(\mathbf{I}+s \mathbf{Z}))} \nabla(\operatorname{det}(\mathrm{D}(\mathbf{I}+s \mathbf{Z}))) \cdot \mathbf{B}^{s} \nabla v^{t, s}\right\} \mathrm{d} \mathbf{x} \mathrm{d} t \\
+ & \frac{1}{s} \int_{0}^{T} \int_{\Omega_{t}}\left\{\left(\nabla v^{t, s}\right)^{\top}(\mathrm{D}(\mathbf{I}+s \mathbf{Z}))^{-1} \partial_{t}(\mathbf{I}+s \mathbf{Z}) \varphi\right\} \mathrm{d} \mathbf{x} \mathrm{d} t,
\end{aligned}
$$

where the last line is new in this time-dependent setting in comparison with the proof given in [2]. We now need to show that $G_{s}$ converges to $G$ stated in (A.8).

Clearly, $\varphi \mapsto G_{s}(\varphi)$ is a bounded linear functional on $\tilde{H}_{0}^{1, \frac{1}{2}}\left(Q_{T}\right)$, i.e. $G_{s} \in$ $\left(\tilde{H}_{0}^{1, \frac{1}{2}}\left(Q_{T}\right)\right)^{\prime}$. Therefore, we can interchange the integration and the limit $s \rightarrow 0$. Especially, as in [2], we have

$$
\frac{1}{s}\left(\mathbf{I}-\mathbf{B}^{s}\right) \rightarrow \mathrm{D} \mathbf{Z}+\mathrm{D} \mathbf{Z}^{\top}
$$

and

$$
\frac{1}{s \operatorname{det}(\mathrm{D}(\mathbf{I}+s \mathbf{Z}))} \nabla(\operatorname{det}(\mathrm{D}(\mathbf{I}+s \mathbf{Z}))) \rightarrow \nabla \operatorname{div} \mathbf{Z}
$$

as $s \rightarrow 0$. Thus, it remains to compute

$$
\lim _{s \rightarrow 0} \frac{1}{s}(\mathrm{D}(\mathbf{I}+s \mathbf{Z}))^{-1} \partial_{t}(\mathbf{I}+s \mathbf{Z}) .
$$


By using the Neumann series, we have

$$
(\mathrm{D}(\mathbf{I}+s \mathbf{Z}))^{-1}=\mathbf{I}-s \mathrm{D} \mathbf{Z}+o(s)
$$

and therefore

$$
\lim _{s \rightarrow 0} \frac{1}{s}(\mathrm{D}(\mathbf{I}+s \mathbf{Z}))^{-1} \partial_{t}(\mathbf{I}+s \mathbf{Z})=\lim _{s \rightarrow 0} \frac{1}{s}(\mathbf{I}-s \mathrm{D} \mathbf{Z}+o(s)) s \partial_{t} \mathbf{Z}=\partial_{t} \mathbf{Z} .
$$

In order to conclude the convergence $G_{s} \rightarrow G$ as $s \rightarrow 0$, we need that $v^{t, s}$ converges to $v$ in $H^{1,0}\left(Q_{T}\right)$. To this end, we transform the equations for $v$ and for $v^{t, s}$ to $Q_{0}$ by using the transformation $\kappa$, yielding two differential equations similar to (A.11). Applying [18, Theorem 4.5 on pg. 166] implies the convergence of $v_{t, s} \circ(\mathbf{I}+s \mathbf{Z}) \circ \boldsymbol{\kappa}$ to $v \circ \boldsymbol{\kappa}$ and thus, with the uniformity condition (2.2), also $v^{t, s}$ converges to $v$. Therefore, we have convergence of $G_{s} \rightarrow G$ as $s \rightarrow 0$ in the dual space of $\tilde{H}_{0}^{1, \frac{1}{2}}\left(Q_{T}\right)$ as in [2], with $G(\varphi)$ as in (A.8).

Now, we can argue as in [2]: since the solution operator is an isomorphism from $\hat{H}^{-1,-\frac{1}{2}}\left(Q_{T}\right)$ to $\hat{H}_{0}^{1, \frac{1}{2}}\left(Q_{T}\right)$ (see Theorem 2.2), the statement in Lemma A.3 is true. $\square$

Having the material derivative for (2.4) at hand, we are finally in the position to prove the local shape derivative posed in Theorem 3.1.

Proof of Theorem 3.1. Starting from the material derivative, we would like to compute the local shape derivative $\delta v$.

If we consider $v \in \hat{H}^{2,1}\left(Q_{T}\right)$, we have $\nabla v \in H^{1, \frac{1}{2}}\left(Q_{T}\right)$ and $\Delta v \in L^{2}\left(Q_{T}\right)$, as in [2]. This follows from $\boldsymbol{\kappa}$ being a diffeomorphism and from the time-independent case in [19, Proposition 2.3 on pg. 14 with $r=2, s=1, j=2$ and $k=0]$. Let us next introduce the test space

$$
V\left(Q_{0}\right):=\left\{v=\left.U\right|_{Q_{0}}: U \in C_{0}^{2}\left((-\infty, T) \times \Omega_{0}\right)\right\},
$$

which is a dense subspace of $\tilde{H}_{0}^{1, \frac{1}{2}}\left(Q_{0}\right)$, compare [2] (for a $C^{\infty}$-boundary, see for example [19, Remark 2.2 on pg. 8]). Likewise to Section 2.2, we define with its help the space $V\left(Q_{T}\right)$, which contains functions $\varphi$, such that $\varphi \circ \kappa \in V\left(Q_{0}\right)$. Then, for $\varphi \in V\left(Q_{T}\right)$, we have the same identity as in [2, pg. 859], namely

$$
\begin{aligned}
\left(\mathrm{D} \mathbf{Z}+\mathrm{D} \mathbf{Z}^{\boldsymbol{\top}}\right) \nabla v \cdot \nabla \varphi+\varphi \nabla(\operatorname{div} \mathbf{Z}) \cdot \nabla v=\operatorname{div}(\operatorname{div}(\varphi \mathbf{Z}) \nabla v-(\nabla v \cdot \nabla \varphi) \mathbf{Z}) \\
+\nabla(\mathbf{Z} \cdot \nabla v) \cdot \nabla \varphi-\operatorname{div}(\varphi \mathbf{Z}) \Delta v .
\end{aligned}
$$

Applying this identity and the divergence theorem to (A.8) yields

$$
G(\varphi)=\int_{0}^{T} \int_{\Omega_{t}}\{\nabla(\mathbf{Z} \cdot \nabla v) \cdot \nabla \varphi-\operatorname{div}(\varphi \mathbf{Z}) \underbrace{\Delta v}_{=\partial_{t} v}+\nabla v \cdot \partial_{t} \mathbf{Z} \varphi\} \mathrm{d} \mathbf{x} \mathrm{d} t
$$

where the boundary terms vanish due to the compact support of $\varphi$. Note that only the last term of the integrand differs from the computations in [2]. It holds

$$
-\partial_{t} v \operatorname{div}(\mathbf{Z} \varphi)=-\operatorname{div}\left(\partial_{t} v \mathbf{Z} \varphi\right)+\mathbf{Z} \varphi \cdot \nabla\left(\partial_{t} v\right)
$$

and, therefore, we can apply the divergence theorem again to get

$$
G(\varphi)=\int_{0}^{T} \int_{\Omega_{t}}\left\{\nabla(\mathbf{Z} \cdot \nabla v) \cdot \nabla \varphi+\mathbf{Z} \varphi \nabla\left(\partial_{t} v\right)+\nabla v \cdot \partial_{t} \mathbf{Z} \varphi\right\} \mathrm{d} \mathbf{x} \mathrm{d} t .
$$


Taking the two time derivatives together yields

$$
G(\varphi)=\int_{0}^{T} \int_{\Omega_{t}}\left\{\partial_{t}(\nabla v \cdot \mathbf{Z}) \varphi+\nabla(\mathbf{Z} \cdot \nabla v) \cdot \nabla \varphi\right\} \mathrm{d} \mathbf{x} \mathrm{d} t
$$

This is the same expression as in [2]. Therefore, we can use the identity (3.3) and follow the rest of the proof in [2, Theorem 2.1]. Thus, the local shape derivative satisfies the same partial differential equation as in [2] except for being in a space-time tube $Q_{T}$ instead a space-time cylinder $Q_{0}$.

\section{REFERENCES}

[1] D. Bucur and G. Buttazzo. Variational Methods in Shape Optimization Problems, volume 65 of Progress in Nonlinear Differential Equations and Their Applications. Birkhäuser, Boston, 2005.

[2] R. Chapko, R. Kress, and J.-R. Yoon. On the numerical solution of an inverse boundary value problem for the heat equation. Inverse Problems, 14(4):853-867, 1998.

[3] R. Chapko, R. Kress, and J.-R. Yoon. An inverse boundary value problem for the heat equation: the neumann condition. Inverse problems, 15(4):1033, 1999.

[4] M. Costabel. Boundary integral operators for the heat equation. Integral Equations and Operator Theory, 13(4):498-552, 1990.

[5] M.C. Delfour and J.-P. Zolésio. Shapes and Geometries: Metric, Analysis, Differential Calculus, and Optimization. Society of Industrial and Applied Mathematics, Philadelphia, PA, second edition, 2011.

[6] J.E. Dennis and R.B. Schnabel. Numerical Methods for Nonlinear Equations and Unconstrained Optimization Techniques. Prentice-Hall, Englewood Cliffs, NJ, 1983.

[7] R. Dziri and J.-P. Zolésio. Dynamical shape control in non-cylindrical Navier-Stokes equations. Journal of Convex Analysis, 6(2):293-318, 1999.

[8] R. Dziri and J.-P. Zolésio. Eulerian derivative for non-cylindrical functionals. In J. Cagol, M.P. Polis, and J.-P. Zolésio, editors, Shape optimization and optimal design, Lecture Notes in Pure and Applied Mathematics, pages 87-107. Marcel Dekker, New York-Basel, 2001.

[9] R. Fletcher. Practical Methods for Optimization. Wiley, New York, 1980.

[10] C. Geiger and C. Kanzow. Numerische Verfahren zur Lösung unrestringierter Optimierungsaufgaben. Springer, Berlin-Heidelberg, 1999.

[11] M.E. Gurtin. An Introduction to Continuum Mechanics. Academic Press, New York, NY, 1981.

[12] H. Harbrecht, M. Peters, and M. Siebenmorgen. Analysis of the domain mapping method for elliptic diffusion problems on random domains. Numerische Mathematik, 134(4):823-856, 2016.

[13] H. Harbrecht and J. Tausch. On the numerical solution of a shape optimization problem for the heat equation. SIAM Journal on Scientific Computing, 35(1):A104-A121, 2013.

[14] M. Hinze, R. Pinnau, M. Ulbrich, and S. Ulbrich. Optimization with PDE constraints, volume 23. Springer Science+Business Media, 2008.

[15] A.D. Ioffe and V.M. Tichomirov. Theorie der Extremalaufgaben. Deutscher Verlag der Wissenschaften, Berlin, 1979.

[16] H. Kawakami and M. Tsuchiya. Uniqueness in shape identification of a time-varying domain and related parabolic equations on non-cylindrical domains. Inverse Problems, 26(12):125007, 2010.

[17] A.M. Khludnev and J. Sokolowski. Modeling and Control in Solid Mechanics, volume 122 of International Series of Numerical Mathematics. Birkhäuser, Basel, 1997.

[18] O.A. Ladyzenskaja, V.A. Solonnikov, and N.N. Ural'Ceva. Linear and Quasilinear Equations of Parabolic Type. American Mathematical Society, Providence, RI, 1968.

[19] J.L. Lions and E. Magenes. Problèmes aux limites non homogènes et applications, volume 2 of Travaux et recherches mathématiques. Dunod, Paris, 1968.

[20] J.L. Lions and E. Magenes. Non-Homogeneous Boundary Value Problems and Applications I. Springer, 1972.

[21] J.L. Lions and E. Magenes. Non-Homogeneous Boundary Value Problems and Applications II. Springer, 1972.

[22] W. McLean. Strongly elliptic systems and boundary integral equations. Cambridge University Press, Cambridge, NY, 2000. 
[23] M. Moubachir and J.-P. Zolésio. Moving Shape Analysis and Control. Chapman \& Hall / CRC, Tayler \& Francis Group, 2006.

[24] J. Nocedal and S.T. Wright. Numerical Optimization. Springer Science+Business Media, LLC, second edition, 2006.

[25] W.H. Press, S.A. Teukolsky, W.T. Vetterling, and B.P. Flannery. Numerical Recipes in FORTRAN 77, volume 1. University of Cambridge, Cambridge, NY, 1992.

[26] J. Sokolowski. Shape sensitivity analysis of boundary optimal control problems for parabolic systems. SIAM Journal on Control and Optimization, 26(4):763-787, 1988.

[27] J. Sokolowski and J.-P. Zolésio. Introduction to Shape Optimization. Springer, BerlinHeidelberg, 1992.

[28] J.H. Spurk and N. Aksel. Fluid Mechanics. Springer, Berlin-Heidelberg, second edition, 2008.

[29] J. Tausch. Nystrom discretization of parabolic boundary integral equations. Applied Numerical Mathematics, 59(11):2843-2856, 2009.

[30] J. Tausch. Nyström method for BEM of the heat equation with moving boundaries. Advances in Computational Mathematics, 45(5-6):953-2968, 2019.

[31] J. Wloka. Partial differential equations. Cambridge University Press, Cambridge, NY, 1987.

[32] S. El Yacoubi and J. Sokolowski. Domain optimization problems for parabolic control systems. Applied Mathematics and Computer Science, 6:277-290, 1996.

[33] J.-P. Zolésio. Identification de domaines par déformations. PhD thesis, Université de Nice, 1979. 